\title{
'We are not going to shut down, because we cannot postpone pregnancy': a mixed-methods study of the provision of maternal healthcare in six referral maternity wards in four sub-Saharan African countries during the COVID-19 pandemic
}

To cite: Semaan A,

Banke-Thomas A, Amongin D, et al. 'We are not going to shut down, because we cannot postpone pregnancy': a mixedmethods study of the provision of maternal healthcare in six referral maternity wards in four sub-Saharan African countries during the COVID-19 pandemic. BMJ Global Health 2022;7:e008063. doi:10.1136/ bmjgh-2021-008063

Handling editor Seye Abimbola

- Additional supplemental material is published online only. To view, please visit the journal online (http://dx.doi.org/10. 1136/bmjgh-2021-008063)

Received 20 November 2021 Accepted 20 January 2022

Check for updates

(c) Author(s) (or their employer(s)) 2022. Re-use permitted under CC BY-NC. No commercial re-use. See rights and permissions. Published by BMJ.

For numbered affiliations see end of article.

Correspondence to

Aline Semaan; asemaan@itg.be

\section{ABSTRACT}

Introduction Referral hospitals in sub-Saharan Africa are located in crowded urban areas, which were often epicentres of the COVID-19 pandemic. This paper prospectively assesses how maternal healthcare was provided in six referral hospitals in Guinea, Nigeria, Tanzania and Uganda during the first year of the COVID-19 pandemic.

Methods Mixed-methods design using three data sources: (1) qualitative data from repeated rounds of semi-structured interviews conducted between July 2020 and February 2021 with 22 maternity skilled heath personnel (SHP) on perceptions of care provision; (2) quantitative monthly routine data on caesarean section and labour induction from March 2019 to February 2021; and (3) timeline data of COVID-19 epidemiology, national and hospital-level events. Qualitative and quantitative data were analysed separately, framed based on timeline analysis, and triangulated during reporting.

Results We identified three periods: first wave, slow period and second wave. The first wave was challenging for SHP given little knowledge about COVID-19, lack of infection prevention and control training, and difficulties reaching workplace. Challenges that persisted beyond the first wave were shortage of personal protective equipment and no rapid testing for women suspected with COVID-19. We noted no change in the proportion of caesarean sections during the pandemic, and a small increase in the proportion of labour inductions. All hospitals arranged isolation areas for women suspected/confirmed with COVID-19 and three hospitals provided care to women with suspected/confirmed COVID-19. Breastfeeding was not discouraged and newborns were not separated

\section{Key questions}

What is already known?

- Restriction measures used to curb the spread of COVID-19 interrupted the provision of materna healthcare services and led to adaptations across the entire continuum of care including antenata care, intrapartum and postnatal care.

- Maternity skilled health personnel (SHP) were at the forefront of implementing regularly updated guidelines and faced many challenges during the COVID-19 pandemic.

- Referral maternity wards in sub-Saharan Africa are mostly located in crowded urban areas which were often epicentres of the COVID-19 pandemic, and some of them assumed the role of healthcare provision for pregnant women suspected or confirmed with COVID-19.

from mothers confirmed with COVID-19. Care provision was maintained through dedication of SHP, support from hospital management and remote communication between SHP.

Conclusion Routine maternal care provision was maintained in referral hospitals, despite first wave challenges. Referral hospitals and SHP contributed to guideline development for pregnant women suspected/ confirmed with COVID-19. Maternity SHP, women and pregnancy must always be included in priority setting when responding to health system shocks, including outbreaks. 


\section{Key questions}

\section{What are the new findings?}

- Maternity wards in referral hospitals and maternity SHP advocated for the inclusion of pregnant women in the planning and response to the COVID-19 pandemic at the national level, including leading and contributing to guideline development and training of lowerlevel facilities.

- Routine care provision was dynamically adapted to include infection prevention and control measures, such as reducing the number of allowed visitors and reducing the length of hospital stay after birth; no changes to indications of caesarean sections and labour inductions were reported.

- Care provision to women suspected/confirmed with COVID-19 was challenging as a result of lack of rapid testing for women in labour, confusion of symptoms of COVID-19 with those of obstetric complications, and lack of guidelines and training.

What do the new findings imply?

- Concrete efforts must be put in place to increase staffing levels in maternity wards, alleviate crowding, make rapid testing for COVID-19 available for women in labour and ensure a sustained supply of protective equipment.

- Essential healthcare services, including maternity care, and frontline maternity SHP must be prioritised in planning and response as the COVID-19 pandemic continues to evolve, and in upcoming healthcare system shocks.

\section{INTRODUCTION}

COVID-19 was declared a pandemic on 11 March 2020 by the World Health Organization (WHO). As of 19 October 2021, over 240 million confirmed cases of COVID-19, and around 5 million deaths, were reported globally. ${ }^{1}$ Out of those, more than 8 million cases and almost 216,000 deaths were reported in Africa. ${ }^{2}$ Governments and health systems struggled globally to respond to the rapid spread of a new disease. In maternity care, evidence regarding the effect of SARS-CoV-2 infection during pregnancy and childbirth was inconclusive for a long period of the pandemic. This uncertainty was accompanied by reorganisation of care provision models and introduction of mitigation measures that were not supported by evidence, such as separation of mothers confirmed with COVID-19 from newborns and not allowing breastfeeding. ${ }^{3} 4$ Currently, there is evidence that women diagnosed with COVID-19 have higher risk of eclampsia/pre-eclampsia, maternal mortality, stillbirth, preterm birth and congenital birth defects. ${ }^{5-9}$ A multinational cohort study showed that delivery by caesarean section, but not breastfeeding, was associated with an increased risk of SARS-CoV-2 transmission to newborns. ${ }^{8}$ The WHO recommends that for women confirmed with COVID-19, labour induction and caesarean section should only be undertaken when medically indicated, and that breastfeeding should be encouraged. ${ }^{10}$

The impact of the pandemic is not limited to affecting women diagnosed with COVID-19. During the early days of the pandemic, between $8.3 \%$ and $38.6 \%$ increase in maternal deaths per month were projected across 118 countries as a result of disruptions in service provision and access. ${ }^{11}$ There is evidence that there have been significant increases in stillbirths and maternal deaths in low- and middle-income countries ${ }^{12}$ mostly driven by COVID-19 restriction measures such as lockdowns and travel bans, which limited the availability of and women's access to quality services. Provision of antenatal and postnatal care was reportedly disrupted in over one-third of countries, while a quarter reported disruptions in facility-based births $(n=121) .{ }^{13}$ Aside from interruptions to care provision, many adaptations were implemented, the implications of which on the quality of care remain unknown. Those include care provision with telemedicine, attempts to minimise the duration women spend in hospitals (either by labour induction or by discharging early after birth), changes in anaesthesia practices and reduction in the number of allowed companions during birth. ${ }^{14-16}$ In an effort to minimise negative consequences, international and national guidelines were developed, modified and adapted for maternal health service provision across the entire continuum of care including antenatal care, intrapartum and postnatal care. ${ }^{17-21}$

Maternity skilled health personnel (SHP)—including doctors, nurses and midwives-were at the forefront of implementing these regularly updated guidelines and faced many challenges during the COVID-19 pandemic. ${ }^{3}$ In a global context of shortage of personal protective equipment (PPE), pre-existing shortage of SHP, and lack of clear information and guidelines, ${ }^{22}{ }^{23}$ maternal and newborn SHP worried about their own health and were concerned over occupational exposure to COVID-19 in the workplace, and transmitting the infection to patients, family and friends. ${ }^{32-27} \mathrm{~A}$ meta-analysis shows that stress disorders, depression, anxiety, and insomnia are highly prevalent among SHP during periods of epidemics and pandemics, including COVID-19. ${ }^{28} \mathrm{~A}$ recent study in Ghana and Kenya shows significant declines in job satisfaction and increase in stress and burnout among frontline healthcare workers during the COVID-19 pandemic. ${ }^{29}$ In Nigeria, maternal and newborn healthcare providers worried about stigmatisation or discrimination as a result of their potential exposure to COVID-19, and 87\% experienced work-related burnout because of the COVID-19 pandemic. ${ }^{26}$

In sub-Saharan Africa, large tertiary referral hospitals are designed to primarily provide care for women with high-risk pregnancies, however they also serve large numbers of low-risk women who prefer to seek care in high-level facilities. ${ }^{30}$ In addition, these referral hospitals take care of the most complicated obstetric cases, provide outpatient and inpatient care to thousands of women and newborns annually, conduct critical research, and train the next generation of SHP, including obstetricians, midwives, and nurses. The majority of these hospitals are located in crowded urban areas with large catchment population. COVID-19 largely spread and was highly concentrated in large cities in many countries, and cities like Lagos, Nairobi and Johannesburg have all 
been the epicentres of their respective countries. ${ }^{2}$ During the COVID-19 pandemic, some of these facilities have assumed the role of healthcare provision for pregnant women suspected or confirmed with COVID-19. ${ }^{32}$ In addition, these facilities have led training, guideline and protocol development and testing of service adaptations in the sub-region.

Townsend et al. document a gap in the literature on maternity care provision during the COVID-19 pandemic in low- and middle-income countries, particularly in subSaharan Africa, ${ }^{16}$ as the majority of the papers published from these contexts quantitatively describe changes in service utilisation. ${ }^{33-37}$ A comprehensive, in-depth, quantitative and qualitative assessment of situation from the perspective of maternal SHP is lacking from the region. This paper is part of a larger study that presents an in-depth description of maternity care in six large maternity referrals in four sub-Saharan African countries (Guinea, Nigeria, Tanzania and Uganda) throughout the first year of the COVID-19 pandemic. The objective of this paper is to prospectively assess the effect of the COVID-19 pandemic on the provision of maternal health services in six large referral hospitals, using a mix of deductive and inductive approaches to allow a flexible and iterative process in exploring the topic at hand.

\section{METHODS \\ Study design}

This study uses a mixed-methods design, relying on three main types of data: qualitative data from key-informant interviews, quantitative routine hospital data, and timeline data of national and hospital-level events and trends during the COVID-19 pandemic. Data from the quantitative and qualitative strands were collected in parallel, analysed separately and framed in light of the timeline analysis. Findings from the qualitative and quantitative strands were triangulated at the reporting stage which allowed for a cross-comparison between the reports of key-informants and the routinely collected statistics. ${ }^{38}$

\section{Hospital selection and profiles}

We purposively selected six hospitals to be included in this study, with a focus on including hospitals with large referral maternity wards in urban areas from four subSaharan African countries (Guinea, Nigeria, Tanzania, Uganda). The selection ensured diversity of healthcare systems in sub-Saharan Africa. Online supplemental table S1 describes in detail the characteristics of each hospital and maternity ward. The participating hospitals are Hôpital National Ignace Deen (HNID) and Hôpital Regional de Mamou (HRM) in Guinea, Lagos University Teaching Hospital (LUTH) in Nigeria, Muhimbili National Hospital (MNH) in Tanzania, Kawempe National Referral Hospital (KNRH) and Mulago Specialised Women's and Neonatal Hospital (MSWNH) in Uganda.

\section{Data}

Timeline data

We captured and summarised key events that could influence service provision at the level of participating hospitals during the period between 1 January 2020 and 28 February 2021, through biweekly team meetings and insights from principal investigators (PIs) and co-PIs. Such events include presentation of the first COVID-19 positive obstetric case, periods of closure of maternity services, opening of COVID-19 isolation centre, modifications in service provision and requirements for patients or their relatives, and deployment of new guidelines. On the national level, we sourced key events from the Oxford COVID-19 Government Response Tracker. ${ }^{39}$ These include periods of national lockdown(s), nighttime curfews, domestic travel bans and mask mandates, among others. The initial data were verified for accuracy and expanded with contextual details by the country teams and through a desk review of weblinks describing the national events. The events were laid over a timeline of the numbers of cases and deaths due to COVID-19 at the national level, collected from 'Our World in Data'40 and validated on the WHO COVID-19 dashboard. ${ }^{1}$ Global events were sourced from the WHO panel.

\section{Routine data}

Monthly aggregated routine health statistics covering the period between 1 January 2019 and 28 February 2021 (24 data points for 24 months) were collected from each of the participating maternity wards. For this analysis, we included two routine indicators representing two aspects of care provision and content of care: caesarean section and labour induction. Indicator definition and data sources by maternity ward are included in online supplemental table S2. When multiple data sources for the same indicator were available, data were collected from all sources and validated against each other. In case of discrepancy, the researchers included values from the most reliable source according to hospital staff and data clerks.

The fieldwork to collect the monthly aggregate data occurred between 1 June 2020 and 28 March 2021, and was conducted by clinical researchers in collaboration with clinicians and data clerks or record officers from the participating hospitals. Aggregated monthly values of indicators were entered into a standardised Excel sheet, and a thorough data review and verification exercise was conducted (at least twice for each hospital). This exercise allowed for detection of outliers and missing values and ensuring data quality and completeness in all six hospitals. We conducted descriptive analysis of each indicator for a period of 24 months, divided into two 12-month time periods: from March 2019 to February 2020 labelled as pre-COVID-19, and from March 2020 to February 2021 labelled as during COVID19. We displayed the results as frequencies and percentages in combined bar and line charts. Changes were explored and compared between the two time periods and were triangulated against findings from qualitative interviews. 


\begin{tabular}{|c|c|c|}
\hline Hospital (country) & Respondent & Number of interviews \\
\hline \multirow[t]{6}{*}{ HNID (Guinea) } & $A$ & 2 \\
\hline & B & 3 \\
\hline & C & 4 \\
\hline & $\mathrm{D}$ & 3 \\
\hline & $E$ & 1 \\
\hline & $\mathrm{F}$ & 1 \\
\hline \multirow[t]{4}{*}{ HRM (Guinea) } & A & 3 \\
\hline & $\mathrm{B}$ & 4 \\
\hline & $\mathrm{C}$ & 4 \\
\hline & $\mathrm{D}$ & 4 \\
\hline \multirow[t]{4}{*}{ LUTH (Nigeria) } & A & 3 \\
\hline & $\mathrm{B}$ & 3 \\
\hline & C & 3 \\
\hline & $\mathrm{D}$ & 1 \\
\hline \multirow[t]{4}{*}{ MNH (Tanzania) } & $A$ & 1 \\
\hline & $\mathrm{B}$ & 3 \\
\hline & $\mathrm{C}$ & 2 \\
\hline & $\mathrm{D}$ & 1 \\
\hline \multirow[t]{2}{*}{ KNRH (Uganda) } & A & 1 \\
\hline & B & 1 \\
\hline \multirow[t]{2}{*}{ MSWNH (Uganda) } & A & 1 \\
\hline & B & 1 \\
\hline Total & 22 & 50 \\
\hline
\end{tabular}

HNID, Hôpital National Ignace Deen; HRM, Hôpital Regional de Mamou; KNRH, Kawempe National Referral Hospital; LUTH, Lagos University Teaching Hospital; MNH, Muhimbili National Hospital; MSWNH, Mulago Specialised Women's and Neonatal Hospital.

\section{Key-informant interviews with maternity SHP}

We conducted semi-structured interviews between July 2020 and February 2021 through repeated rounds of data collection with two to six maternity SHP in each maternity ward. Each hospital's PI suggested a list of potential key-informants, purposively selected to ensure maximum variation in the experiences of SHP, including from various seniority levels (junior and senior staff), cadres (medical doctors, midwives and nurses) and genders. If the selected SHP expressed interest in participating, their contact information was shared with ND and LB. The researchers randomly selected some of the keyinformants on the list, and invited them to participate in the interview. Informed consent was administered by ND and LB for respondents in Guinea, Nigeria and Tanzania, and by the PI in Uganda. Recruitment stopped when data saturation was reached for each round of data collection. Respondents were compensated for their time and use of mobile data after each interview. In total, 22 SHP were interviewed, and 50 interviews took place (table 1).

We used a semi-structured interview guide, allowing us to consistently and comprehensively capture changes in the processes of care provision across all hospitals over the study period. The guide included questions and probes on SHP's perceptions of changes in care inputs (staff, equipment, supplies, beds, training, information materials), care processes (e.g. which services are available, how the wards are organised), and any observations on effectiveness of measures taken to prepare or care for women and newborns with COVID-19. Interviews were conducted by two researchers virtually using Zoom for Nigeria, Tanzania and Uganda (LB), and face-to-face in Guinea (ND). Interview duration ranged between 20 and $120 \mathrm{~min}$. Interviews were recorded and transcribed in the language of the interview (English or French languages), de-identified using a coding scheme and imported into the computer-assisted qualitative data analysis software Dedoose. Analysing the qualitative data was an iterative process and started concurrently with data collection allowing the researchers to adapt the interview guide in the repeated interview rounds based on respondents' answers to the previous rounds and to the country situation.

Data analysis was conducted using the framework method which belongs to the family of qualitative content analysis. ${ }^{41}$ Three researchers (ND, AS and LB) familiarised themselves with the data by re-listening to the recordings and reading the transcripts. The researchers then coded independently six interviews from which new codes and themes were identified (inductive analysis) keeping the structure of the interview guide in mind (deductive). Codes were grouped in categories into a tree diagram (with parent codes and child codes up to three levels). The coding tree was refined during the analysis by adding, merging and deleting codes as appropriate. The coding tree was applied to the 50 interviews systematically by one researcher and checked by another (ND, AS, LB). We mapped the themes and examples emerging from these interviews into a matrix by period (first wave, slow period, second wave-table 2) and by three key dimensions (healthcare worker experiences, changes in the provision of routine maternity care and care for

Table 2 Identified time periods and their respective dates per country

\begin{tabular}{llll}
\hline Country & First wave & Slow period $^{*}$ & Second wave* $^{*}$ \\
\hline Guinea & From March to August 2020 & From September 2020 to January 2021 & -- \\
Nigeria & From March to June 2020 & From July to October 2020 & From November 2020 to January 2021 \\
Tanzania & From March to June 2020 & From July to November 2020 & From December 2020 to January 2021 \\
Uganda & From March to June 2020 & From July to September 2020 & From October 2020 to January 2021 \\
\hline
\end{tabular}

*The last time periods' end date was selected as the end of the study period: January 2021. 
women confirmed with COVID-19, that is, women who had a positive PCR test result during their stay in the hospital) separately for each maternity ward. These extensive notes in the matrix were shared with hospital PIs to confirm objective statements related to the timeline, for example, dates of service closures or introduction of new guidelines. The themes were further summarised to capture similarities and differences across the three time periods and six hospitals, and to identify relationships between the main themes and sub-themes in the data. Illustrative quotes were added to the results to give examples of the themes and sub-themes in the data; respondents are referred to by randomly assigned codes for confidentiality purposes.

\section{Triangulation and synthesis}

This research project was based on principles of stakeholder analysis, self-reflection, supportive cross-country learning and support to junior researchers in the team. The triangulation and synthesis of data from the three data sources was conducted in an iterative, prospective and collaborative manner, by sharing and discussing findings during 21 biweekly research team meetings between May 2020 and March 2021, and upon completion of all data collection in April-July 2021. Triangulation involved summarising the qualitative and quantitative data by the three identified time periods. Time trends observed in the routine data indicators were compared against findings from the qualitative data, including SHP's perceptions and views on content of care provision, to identify and discuss alignment and/or differences between the two data sources over time.

\section{RESULTS}

We identified three time periods based on the timeline analysis with key events, the epidemiological data described by Salyer $e t a t^{42}$ and through regular communication with country teams (table 2). The results of the key-informant interviews and the routine data are reported in an integrated manner against the three identified time periods, starting with a description of the key events related to care provision by the three identified time periods (figure 1).

\section{Timeline of key hospital, national and global events}

The extracted national timeline data show that in Guinea, the first wave extended from March to August 2020, when national restriction measures were initiated, including travel restrictions, night-time curfew, domestic travel ban and bans on mass gatherings. At the hospital levels, staff were reorganised into 24-hour teams and interns were put on mandatory leave since 26 March 2020. In HNID (Guinea), all team communications were moved to WhatsApp; and in HRM (Guinea), a COVID-19 response committee was established. During the slow period, from July to January 2020, a COVID-19 treatment centre opened in Mamou, and the first obstetric case with COVID-19 was received at HNID (Guinea). Interns returned from the mandatory leave in November 2020 in HRM, and in December 2020 in HNID.
In Nigeria, the first wave extended from 30 March 2020 until July 2020. During this period, a night-time curfew, school closures, mandate on face masks in public and a ban on gatherings of more than 50 people were instituted at the national level. The Society of Gynaecology and Obstetrics of Nigeria recommended guidelines for service provision on 27 March 2020. At the hospital level, antenatal care started to be provided as a hybrid model (in-person and telemedicine consultations) as of 23 March 2020, and the maternity service closed between 6 May and 1 June 2020 due to shortage in maternity SHP following diagnosis with COVID-19. A LUTH (Nigeria) isolation centre started operating from 7 April and the first obstetric case with COVID-19 in LUTH (Nigeria) was received on 25 April 2020. The slow period extended between July and October 2020, during which only a night-time curfew and mask mandate continued to be applied. The second wave started in November 2020, with a sharp rise in the number of COVID-19 cases in January nationally.

In Tanzania, the first wave extended between March and June 2020. During this period, schools closed, there was a ban on mass gatherings and travel restrictions were applied at the national level. In MNH (Tanzania), antenatal care visits were spaced for women of low-risk, an isolation centre opened, and limitations were applied to hospital visitations by relatives and attendance of health worker meetings. The first obstetric COVID-19 case was received at MNH (Tanzania) on 13 April 2020. By 4 July 2020, the government declared that the pandemic was over in Tanzania indicating the start of the slow period. No new confirmed cases of COVID-19 were added to the country total of 509 after this. In December 2020, we noted from the country team and respondents the beginning of an increase in the spread of the virus nationally, indicating the beginning of the second (undeclared) wave, when no national restriction measures were witnessed.

In Uganda, the first wave extended between March and June 2020, a period during which a night-time curfew was instituted. This period also witnessed the implementation of school closures, mask wearing mandate, travel restrictions, ban on mass gatherings and domestic travel bans. At the level of the hospitals, guidelines limited the number of relatives allowed to visit to one, outpatient clinics closed, and elective surgeries were limited or stopped. The first obstetric case with COVID-19 was diagnosed on 12 April 2020. The national-level mitigation measures continued to be implemented during the slow period, between July and September 2020, except for the curfew. In October 2020, the number of COVID-19 cases nationally started to rise, indicating the beginning of the second wave, with a peak reached in December 2020. During this period, two floors at MSWNH (Uganda) were dedicated strictly to COVID-19 treatment.

On 1 June 2020, the WHO issued guidance on maintaining essential services, including maternal and newborn services. 


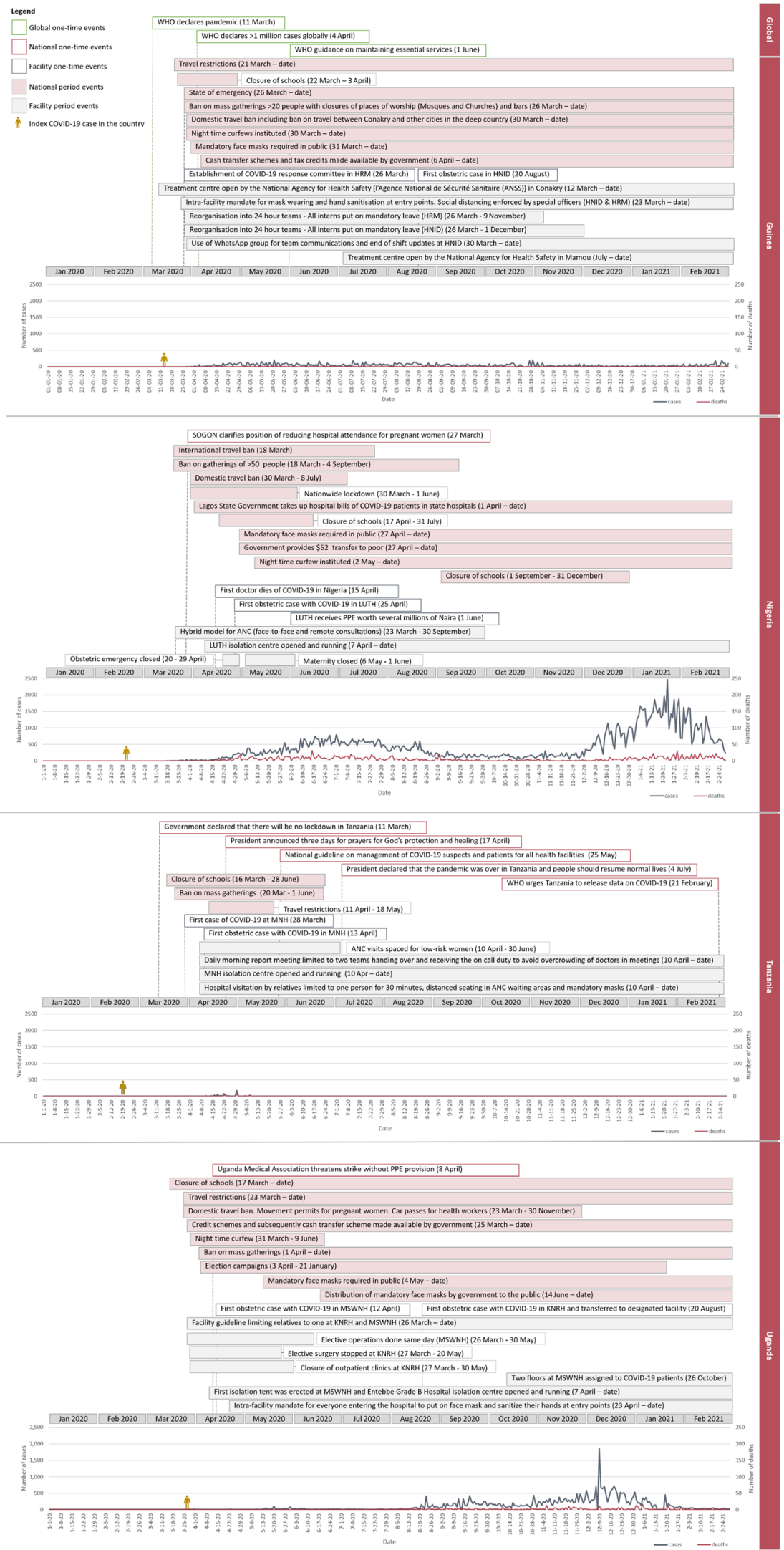

Figure 1 Timeline of key events and COVID-19-confirmed cases and deaths in Guinea,Nigeria, Tanzania and Uganda. ANC, antenatal care; HNID, Hôpital National Ignace Deen; HRM, Hôpital Regional de Mamou; KNRH, Kawempe National Referral Hospital; LUTH, Lagos University Teaching Hospital; MNH, Muhimbili National Hospital; MSWNH, Mulago Specialised Women's and Neonatal Hospital; PPE, personal protective equipment; SOGON, Society of Gynaecology and Obstetrics of Nigeria. 
The results from the interviews and the analysis of routine data are reported with illustrative quotes and/or numbers and figures. The findings are summarised into three main themes: SHP's experiences; changes in provision of routine maternal care and caring for women with COVID-19.

\section{Maternity SHP's experiences with care provision}

Three sub-themes were identified during the first wave. First, SHP reported being stressed and fearful, predominantly due to lack of knowledge about the transmission of the new virus and best ways to protect themselves from infection on the job and consequently protect their families. Respondent B in LUTH (Nigeria) expressed: 'the first thing that happened was fear, until the hospital started training us and we got an understanding of the disease condition. Before [that] we were all scared.' In Uganda, respondents additionally reported being negatively affected by what they described as a shock from the unexpected total lockdown. The second theme, closely linked to stress and fear, was related to working in an environment with a shortage of COVID-19-related PPE (especially high-grade face masks and hand sanitisers), not knowing which type of PPE was needed in different clinical encounters and having to purchase own PPE. Third, respondents described severe, often pre-existing, staff shortages in maternity wards which were exacerbated during COVID-19 due to infections and exposure among SHP, inability to travel during lockdown and lack of help from medical interns following their exclusion from staff rosters. Within this theme, respondents highlighted how staff went above and beyond their duty to continue providing maternity care; examples included sleeping in hospitals to ensure sufficient staffing or volunteering to provide care to COVID-19-positive pregnant women: 'despite the fact that there was hardship in travelling to come and reach work, the staff at Kawempe worked willingly. Some of them even slept over and woke up in the morning and continued work [...]' (Respondent A, KNRH).

In the slow period and the second wave, respondents noted that they felt less stressed than during the first wave of COVID-19. This was related to improved availability of knowledge on SARS-CoV-2 transmission, and more clarity on the protective measures needed, especially after receiving training and protocols relevant to PPE use and rationing in most hospitals. In some hospitals, the improved availability of PPE, including as a result of transferring additional cost of PPE onto patients, also contributed to relieving stress among respondents. Nonetheless, issues with PPE availability continued during the slow period and second wave as Respondent B from MNH (Tanzania) explained during the slow period: 'Even now, we still don't have enough protective equipment. [...] they give you three face masks but you have to use them for one month, three for one month!' Additionally, SHP worried about relaxation of uptake of protective measures on the community level (opening of churches, crowding in public transport, etc.) and lax adherence to rules on wearing masks and hand hygiene among patients and visitors in the hospitals. This worry escalated in the second wave when COVID-19 cases were increasing in several countries, and new variants started to emerge globally. In Tanzania, respondents were reporting fear due to increase in the number of patients with COVID-19-like symptoms in the hospital while on the country level, it was impossible to understand the epidemic situation: 'in this country, it [COVID19] has not been taken as a serious problem, so, people are just. . life as usual' (Respondent C, MNH).

Across all three periods and all maternity wards, we noted several differences in the experiences by cadre. For example, midwives in two Uganda hospitals did not receive a sufficient number of travel stickers to enable movement during lockdown compared with doctors; lower availability of PPE in the second and third time period was noted by midwives compared with doctors in MNH (Tanzania) and LUTH (Nigeria); and changes in staff shift duration to 24 hours in Guinea effectively resulted in only midwives being on duty at night while doctors went home.

\section{Changes in provision of routine maternity care}

Despite a few interruptions to service provision during the first wave, all hospitals continued to provide routine maternal care throughout the study period. This was made possible by the implementation of infection prevention and control (IPC) measures, and by adopting a set of adaptations and changes to pre-existing guidelines and protocols, to staffing and communication, and to care availability and content. Respondent D from Nigeria explained: 'when the pandemic started, almost all outpatient clinics of the hospitals were shut down, except the maternity service. We were the only people who said 'We are not going to shut down because we cannot postpone pregnancy' and some of these women, we have cared for them from the beginning, we cannot just turn our back on them. [...] but we had to modify our protocol and try to make the working environment safer.

\section{Infection prevention and control}

Respondents from Guinea, Nigeria and Tanzania indicated that many of the IPC measures that were implemented, and the preparedness related to preventing the spread of the infection, were inspired by lessons learnt from previous responses to Ebola virus disease (EVD) outbreaks. This included retraining the same personnel responsible of IPC, and the re-establishment of the same infectious disease committees in HRM (Guinea), LUTH (Nigeria) and MNH (Tanzania). In HNID (Guinea), hand washing stations that were functional during EVD outbreaks were reactivated as of the first wave.

All hospitals had initially established a temperature screening station at the main gate and maternity ward entry, required patients and their attendants to wear masks, and introduced strict rules on hand hygiene either by washing hands or using disinfecting solution. Many of the adaptations related to hand hygiene were highly appreciated by respondents and considered 
as positive changes to potentially keep implementing even after the end of the COVID-19 pandemic, such as the installation of more hand washing stations closer to care points and with elbow closing. Nonetheless, these measures and patients' and visitors' compliance reportedly declined in the slow period. In the second wave, it was difficult to reintroduce these precautionary measures with increasing patient numbers, particularly in Tanzania considering the government's declaration that there is no COVID-19 in the country as of July 2020. A SHP in $\mathrm{MNH}$ (Tanzania) explained: If you come right now to the ward, you won't see anything special, you won't see any changes, the wards are full, women are there, they are not even wearing their masks, they don't even want to wear their masks sometimes' (Respondent C, MNH).

Additionally, it was challenging for SHP to provide care with implementing distancing measures and spacing of beds and separating patients from one another, as hospitals suffered from crowding and lack of space availability even before the pandemic. Space constraints continued to exist and became serious concerns during the pandemic as they made physical distancing difficult to implement in practice: 'The guidelines are not hard to put in practice but the situation you are in, is what makes it difficult. [...] [reducing the number of labouring women in the ward] is still hard because of the lack of space we have at the moment' (Respondent A, KNRH).

\section{Guidelines and protocols}

Adaptations to guidelines and protocols were reported in most hospitals throughout the three periods, and SHP mostly struggled with the applicability of these changes in resource-limited contexts. Care protocols before the COVID-19 pandemic did not allow birth companions in the maternity ward due to privacy issues, yet having visitors in inpatient wards was necessary to support women, bring them food and medication, clean laundry, etc. During the first wave, in all hospitals except in MNH (Tanzania), the number of allowed visitors in the postnatal period was reduced to one person as it was not possible to ban visitors altogether considering that they provide essential amenities to women during their hospital stay. These restrictions were lifted during the slow period and there were attempts to reintroduce them during the second wave. In MNH (Tanzania), reducing the number of visitors to two was gradually implemented in January 2021, which was further reduced to one visitor in February 2021; relatively late compared with the other hospitals. In all hospitals, SHP reported difficulties in enforcing the one-visitor rule, such as in Guinea due to cultural norms: 'This [crowding due to visitors] is one of the big problems of the service because people have the culture of coming in large numbers with the patients and people tend not to take the disease [COVID-19] into account in order to change this mentality. You still see 3 to 4 [visitors], so it's hard for us' (Respondent D, HNID).

Before the COVID-19 pandemic, postnatal length of stay was relatively short to alleviate crowding, ranging between 6 and 24 hours after a vaginal birth (table 1). During the study period, SHP reported that shortening the length of stay in hospitals after childbirth was not officially implemented as a change in guidelines and protocols and was not systematically applied. However, length of stay might have effectively reduced if the wards were crowded or upon women's request to leave the hospital early in fear of becoming infected with the virus: Before COVID-19, we tend to do 48 hours because the paediatricians want to do serum bilirubin on the baby before the woman goes home. Serum bilirubin is done 48 hours after birth, but now they go home [after] 24 hours, they can come from home to do it, or they teach them how to check for it once you see a sign of it just come' (Respondent C, LUTH).

\section{Staffing and team communication}

Respondents in all hospitals reported changes in maternity ward staffing patterns during the first wave. These adaptations included changes in the number and type of cadres per shift and in shift duration to reduce number of staff on duty at any given point and to avoid contacts between different teams. Another factor that contributed to the reduction in the number of staff was the reassignment of maternity SHP to COVID-19 treatment centres in MNH (Tanzania) during the first wave: 'when COVID-19 was at its peak, most of the healthcare workers were taken from different wards and stationed in places where there were confirmed cases of COVID-19. [...] In the end there were only a few health workers left who had now to take care of the many patients who are not COVID-19' (Respondent $\mathrm{B}$, $\mathrm{MNH}$ ). Additionally, the banning of medical students or interns from most hospitals contributed to staff shortage during the first wave and extended to the slow period in some hospitals.

The continuity of implementation of these measures over time varied by hospital. Some of these measures were relaxed in the slow period but remained in place and were reintroduced during the second wave. In Uganda, shortage of staffing was reported in the second wave because of lack of medical students/interns, and because of staff having to isolate: 'That is a stressful moment around the hospital now, because some staff get tested and are positive and have to stay off work more than 14 days [...] and we are facing hardship covering for this. It is very hard, but it is happening' (Respondent A, KNRH).

On the other hand, respondents from Guinea reported that teaching and training of medical students and interns had to resume as soon as the situation allowed, in order to prevent the exacerbation of pre-existing shortages in SHP in the country: 'There are certain measures that need to be taken to adapt to the situation so as not to hinder the functioning of the hospital. We have lost a year of teaching of specialists, Guinea needs gynaecologists, we need to train them' (Respondent C, HNID). The return of interns was perceived positively by SHP who reported starting to feel exhausted before interns were allowed to return to maternity wards. 
Clinical team communication transitioned to virtual communication, predominantly WhatsApp group chats, during the first wave, and remained partly on this basis over the whole study period. This was most prominent in LUTH (Nigeria), where even maternal death review committee meetings were conducted virtually: 'WhatsApp groups include all the doctors, all the nurses, the cleaners and the theatre technicians are part of $i$. Apart from the group one, we have sub-groups. [...] it is very very helpful, and it's cost effective compared to trying to call, and the beauty of it, you can call in the poor network, once the person gets the [message] and even if it's late they can respond' (Respondent D, LUTH). Virtual communication was least used in MNH (Tanzania), where respondents reported that staff meetings continued to take place in person. Respondents reported that they were not reimbursed for the additional cost of working by accessing mobile data, and this was particularly noted as financially burdensome by nurses and midwives. Still, many respondents in Guinea mentioned that this virtual mode of communication was practical and worth continuing beyond COVID-19.

\section{Care availability and modality}

During the first wave, the provision of outpatient antenatal care in LUTH (Nigeria) and KNRH (Uganda) was severely affected by clinic closures. In order to reduce crowding in clinics, hospitals instituted longer time periods between antenatal visits, and/or replaced some outpatient care visits (antenatal, postnatal) with telemedicine appointments, such as in LUTH (Nigeria). The hybrid model of telemedicine and in-person antenatal care was appreciated by SHP in LUTH (Nigeria) as a positive adaptation that can be carried forward beyond the pandemic. Whereas respondents from hospitals in Guinea and Uganda reported not using telemedicine due to lack of connectivity, low levels of literacy among patients or lack of reimbursement to SHP of associated telecommunication costs. Respondents in several hospitals also commented that the quality of in-person consultations with women during the first wave was sub-optimal as health workers attempted to keep distance and shorten the duration of the interaction; for example, by asking about fetal movements as opposed to measuring fetal heart rate with a Pinard stethoscope: '[...] 'Madam is your baby still moving, can you perceive the movement of the baby?' The woman says 'yes'. They just document that the baby is fine. And we know that that is not enough' (Respondent C, LUTH).

In most hospitals, the provision of inpatient care was restricted to emergency cases, and all elective obstetric procedures were suspended during the first wave. In $\mathrm{MNH}$ (Tanzania), referrals were rerouted and transferred to another facility during the first wave. However, the decision to suspend elective surgeries was not officially supported by the hospital's management considering the denial of the disease at the national level. SHP took the matter in their own hands as they informally tried to reduce the number of elective surgeries booked in the operating theatre, given the risky environment in the theatre that promotes the spread of infection (closed space with bad ventilation). In Uganda, the availability of maternity care could have been compromised by the national health authorities' proposal of designating MSWNH (Uganda) as a COVID-19 treatment centre. Nonetheless, the hospital's management successfully negotiated that the hospital keeps its original function of providing care to women, and only dedicated two floors for the management of COVID-19 patients as of November 2020.

\section{Routine care provided to women without COVID-19}

Regarding the provision of routine care, respondents did not report any changes to the indications for caesarean section and labour induction. In the routine data, the proportion of caesarean sections was similar during the pandemic compared with the previous year in most hospitals, except for some increases noted in MSWNH (Uganda) during the majority of the pandemic period, and in MNH (Tanzania) in November 2020 (figure 2). The percentage of labour inductions out of all deliveries was highly variable month to month in all hospitals and did not exceed 7\% in HNID and HRM (Guinea) but rose up to $14 \%-16 \%$ in LUTH (Nigeria) and $\mathrm{MNH}$ (Tanzania) (figure 3). Overall, a small increase in the proportion of labours induced was noted in most hospitals during the pandemic; in HNID (Guinea) starting in June 2020; in HRM (Guinea) as of November 2020; in LUTH (Nigeria) between May and November 2020; and in MNH (Tanzania) between March and August 2020. In Uganda, the proportion of labours induced was relatively constant throughout both years in KNRH (Uganda), and data were not available from MSWNH (Uganda).

\section{Caring for women with COVID-19}

Three of the hospitals participating in our study provided care to pregnant women suspected or confirmed with COVID-19 (table 3). The total number of confirmed cases ranged from 4 in MSWNH (Uganda), 5 in $\mathrm{MNH}$ (Tanzania) and 23 in LUTH (Nigeria) during the study period. From the interviews, five subthemes were mentioned and discussed by SHP: organisation of space; planning and coordination of hospital roles; symptom and case identification; lack of specific guidelines and training; and content of care; these are illustrated using quotes in online supplemental table S3.

\section{Organisation of space}

Despite an acute pre-COVID-19 lack of space and crowding, respondents described that all hospitals managed to set-up an isolation space during the first wave, whether it was a separate room or a curtained off area within the maternity ward. These isolation spaces had been used and retained throughout the study period in all hospitals except for MNH (Tanzania), where it was temporarily discontinued in the slow period and reinstated in another location during the second wave. 


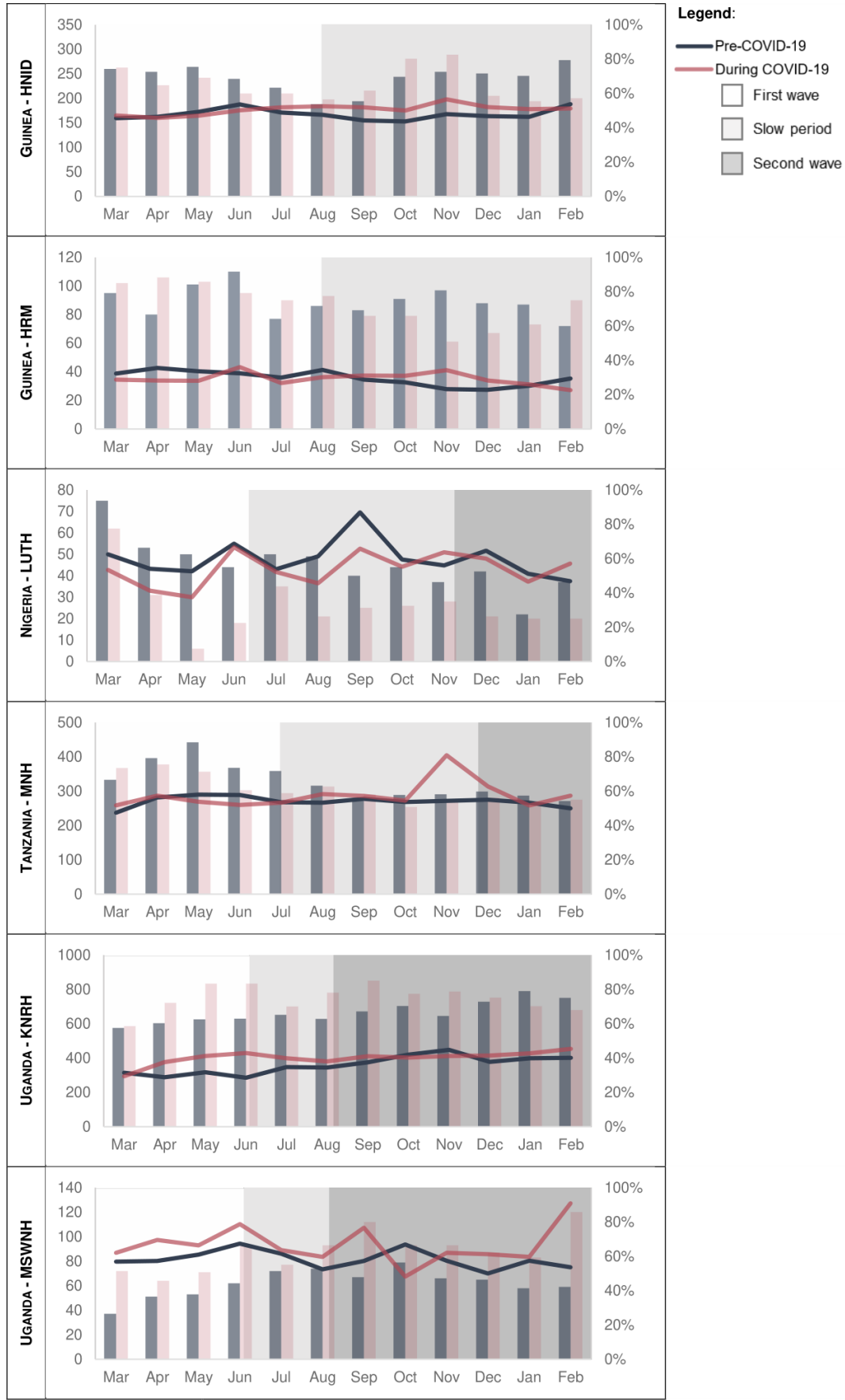

Figure 2 Number (left y axis - bars) and percentage (right y axis - lines) of caesarean sections out of all deliveries by month in each referral hospital before and during the COVID-19 pandemic. HNID, Hôpital National lgnace Deen; HRM, Hôpital Regional de Mamou; KNRH, Kawempe National Referral Hospital; LUTH, Lagos University Teaching Hospital; MNH, Muhimbili National Hospital; MSWNH, Mulago Specialised Women's and Neonatal Hospital.

\section{Planning and coordination of hospital roles}

During the first wave, respondents highlighted the importance of the maternity team's preparedness for the first case of a woman admitted with suspected or confirmed COVID-19 infection, and the processes of care surrounding this case. This was complicated by the fact that in several settings, pregnant women with COVID-19 were not considered in the preparation plans, in terms of specialised staff and equipment in the dedicated
COVID-19 treatment centres; but neither were they expected to be cared for in regular maternity wards (e.g., no negative pressure operating theatres, insufficient staffing, no COVID-19 training to obstetric SHP). This gap resulted in somewhat last-minute planning and accommodation when a first case was admitted, and reluctance of SHP, even those who initially volunteered for COVID-19 teams, to provide care to women with COVID-19 symptoms. Respondents mentioned that receiving support 


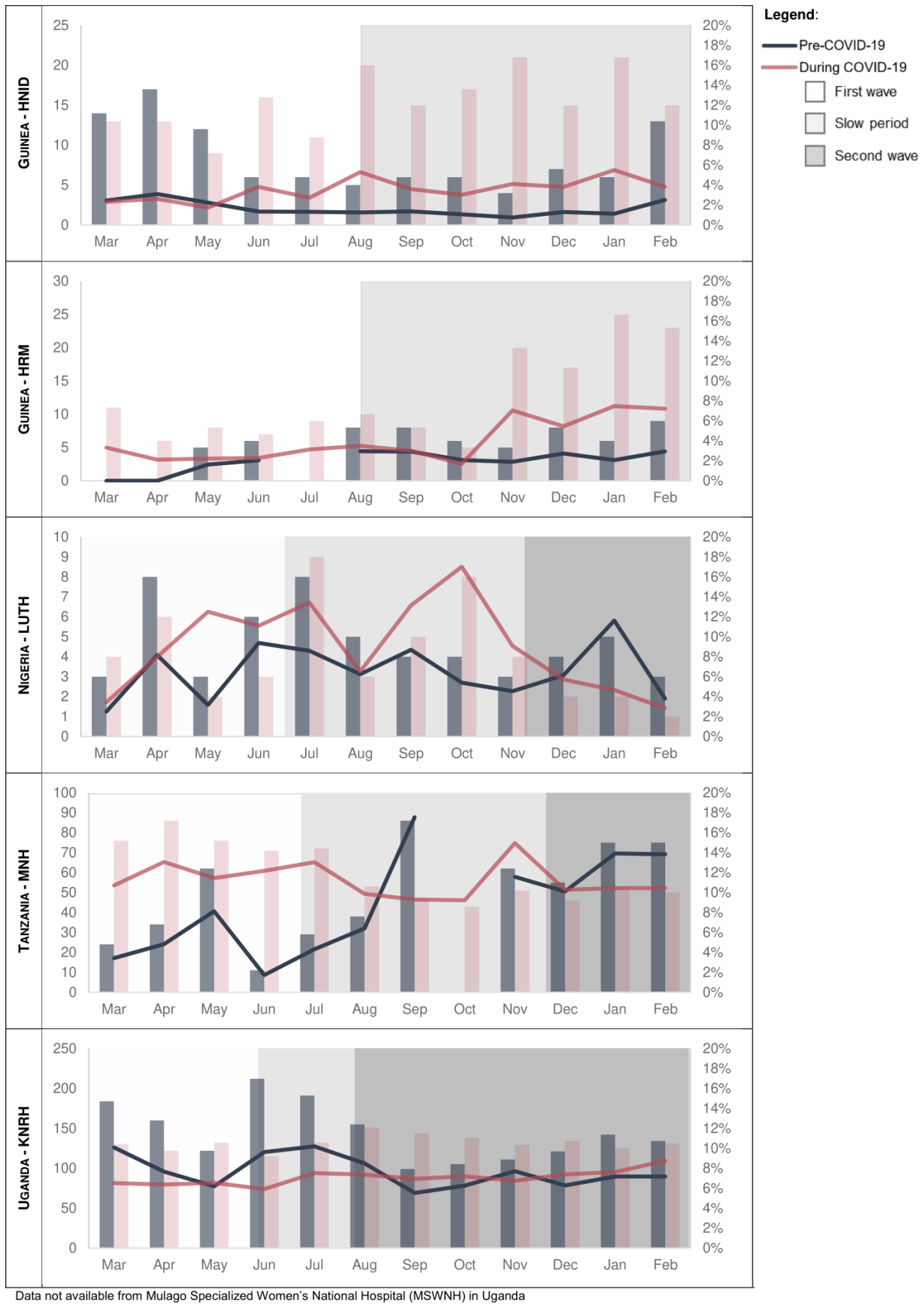

Figure 3 Number (left y axis - bars) and percentage (right y axis - lines) of labour inductions performed out of all deliveries by month in each referral hospital before and during the COVID-19 pandemic. HNID, Hôpital National Ignace Deen; HRM, Hôpital Regional de Mamou; KNRH, Kawempe National Referral Hospital; LUTH, Lagos University Teaching Hospital; MNH, Muhimbili National Hospital.

from the hospital management enabled preparedness for and care provision to the first COVID-19 obstetric case. For example, in LUTH (Nigeria), a member of the team caring for the first obstetric COVID-19 case successfully communicated and advocated for the team's needs: 'in a short while [the hospital management] were able to fix whatever we needed them to fix in the theatre, they got us every kind of PPE we wanted, just to give confidence to the team' (Respondent D, LUTH).
Challenges related to linkages to other health facilities and organisation of the referral system for women with confirmed COVID-19 were frequently mentioned by respondents. Respondents reported being confused about where women with COVID-19 symptoms should be referred and the role played by COVID-19 treatment centres in care for pregnant and postpartum women. This was discussed in light of the absence of obstetric teams in COVID-19 treatment centres in case a woman suspected 
Table 3 Total number of women with suspected or confirmed COVID-19 from March 2020 to February 2021 per hospital

$\begin{array}{ll}\begin{array}{l}\text { Number of women } \\ \text { with suspected }\end{array} & \begin{array}{l}\text { Number of women } \\ \text { with confirmed }\end{array} \\ \text { COVID-19 } & \text { COVID-19 }\end{array}$

\begin{tabular}{lll}
\hline HNID (Guinea) & 0 & 0 \\
HRM (Guinea) & 0 & 0 \\
LUTH (Nigeria) & 6 & 23 \\
MNH (Tanzania) & 50 & $5^{\star}$ \\
KNRH (Uganda) & 0 & 0 \\
MSWNH & 13 & 4 \\
(Uganda) & & \\
\hline
\end{tabular}

* All five cases were recorded before May 2020

HNID, Hôpital National Ignace Deen; HRM, Hôpital Regional de Mamou; KNRH, Kawempe National Referral Hospital; LUTH, Lagos University Teaching Hospital; MNH, Muhimbili National Hospital; MSWNH, Mulago Specialised Women's and Neonatal Hospital.

or confirmed with COVID-19 is imminently giving birth or suffering from an obstetric emergency. According to our respondents, this system requires efficient communication and coordination between hospitals, which was not always the case. This element seems to have improved somewhat over the study period, with clarifications on roles of hospitals and teams, processes, transfers, referrals; although this was continuing to occur in a context of critical shortages of staff, ambulances, space and oxygen.

\section{Symptom and case identification}

Two topics about women suspected with COVID-19 were mentioned by respondents in all three time periods of this study. First, in day-to-day work, they struggled with the difficulty in distinguishing symptoms such as fever, difficulty breathing and cough, which are similar between obstetric complications (e.g., pulmonary embolism, postpartum sepsis) and other common illnesses (e.g., malaria) and COVID-19. Second, in situations where a woman with such non-specific symptoms was admitted in maternity wards, SHP noted that lack of rapid testing for COVID-19 during the entire study period in all hospitals (24-48 hours of waiting times for test results were reported) contributed to increased potential exposure to COVID-19 among SHP and other patients and caused critical delays in providing care to women.

\section{Lack of guidelines, support and training}

Health workers highlighted the importance of having clear guidelines in place for the provision of care to women with suspected/confirmed COVID-19. This was achieved completely in LUTH (Nigeria) by the second wave, with regular updates being included in the guidelines and communicated to SHP, according to international recommendations. In Guinea, the hospitals regularly received updated guidelines from the Ministry of Health, but no official training on the management of COVID-19 obstetric cases was conducted. In MNH (Tanzania), guidelines were developed but not officially approved, this was a key gap noted by respondents, and resulted in women with COVID-19 symptoms receiving care in the same spaces and by same staff as those without suspected COVID-19: 'we still have some dilemmas at the time of management, like when you see a pregnant woman [...], is it COVID-19 patient? And we don't know at what time you have to deliver the woman and so forth. So, sometimes, in terms of guideline, I think we have not reached to that point yet' (Respondent $\mathrm{D}, \mathrm{MNH})$.

\section{Content of care}

In terms of content of care to women confirmed with COVID-19 and their babies, none of the respondents reported that breastfeeding was discouraged, or that newborns were separated from their mothers, unless the mothers were extremely unwell. Respondents in HNID (Guinea) reported augmenting or speeding up labour among women with COVID-19 symptoms, and respondents at LUTH (Nigeria) commented that most women with COVID-19 gave birth by a caesarean section but this was required by the women's clinical condition rather than a result of trying to shorten labour or duration spent in the maternity ward.

\section{Synthesis of findings on maternity care provision during the COVID-19 pandemic: challenges, facilitators and care provision over time}

Figure 4 summarises the themes and sub-themes identified in the interviews and routine data analysis and displays them by study period, highlighting the extent and nature of challenges that maternity SHP faced and the adaptations and strengths that enabled care provision to continue. Overarching themes that were mentioned in most of the referral hospitals are included in the figure, as well as some themes which are specific to certain hospitals/countries. The name of the hospital and/or country is specified in parentheses next to each of these countryspecific themes. The majority of challenges and barriers appeared during the first wave and were overcome as the slow period began. These 'short-term' challenges include maternity SHP's lack of knowledge about COVID-19, lack of training on IPC and inability to reach the workplace, among others. Other challenges persisted during the slow period and/or second wave, such as lack of PPE, lack of rapid testing for women suspected with COVID-19 and difficulties in distinguishing COVID-19 symptoms from symptoms of obstetric complications. Two challenges appeared in the slow period and continued during the second wave, and those were linked to a decline in the national-level and community-level commitment to acknowledge the pandemic and actively prevent its spread. Noteworthy, challenges that maternity wards faced before the COVID-19 pandemic, such as shortage in SHP and crowding in maternity wards, were exacerbated throughout the study period and negatively affected care processes. 


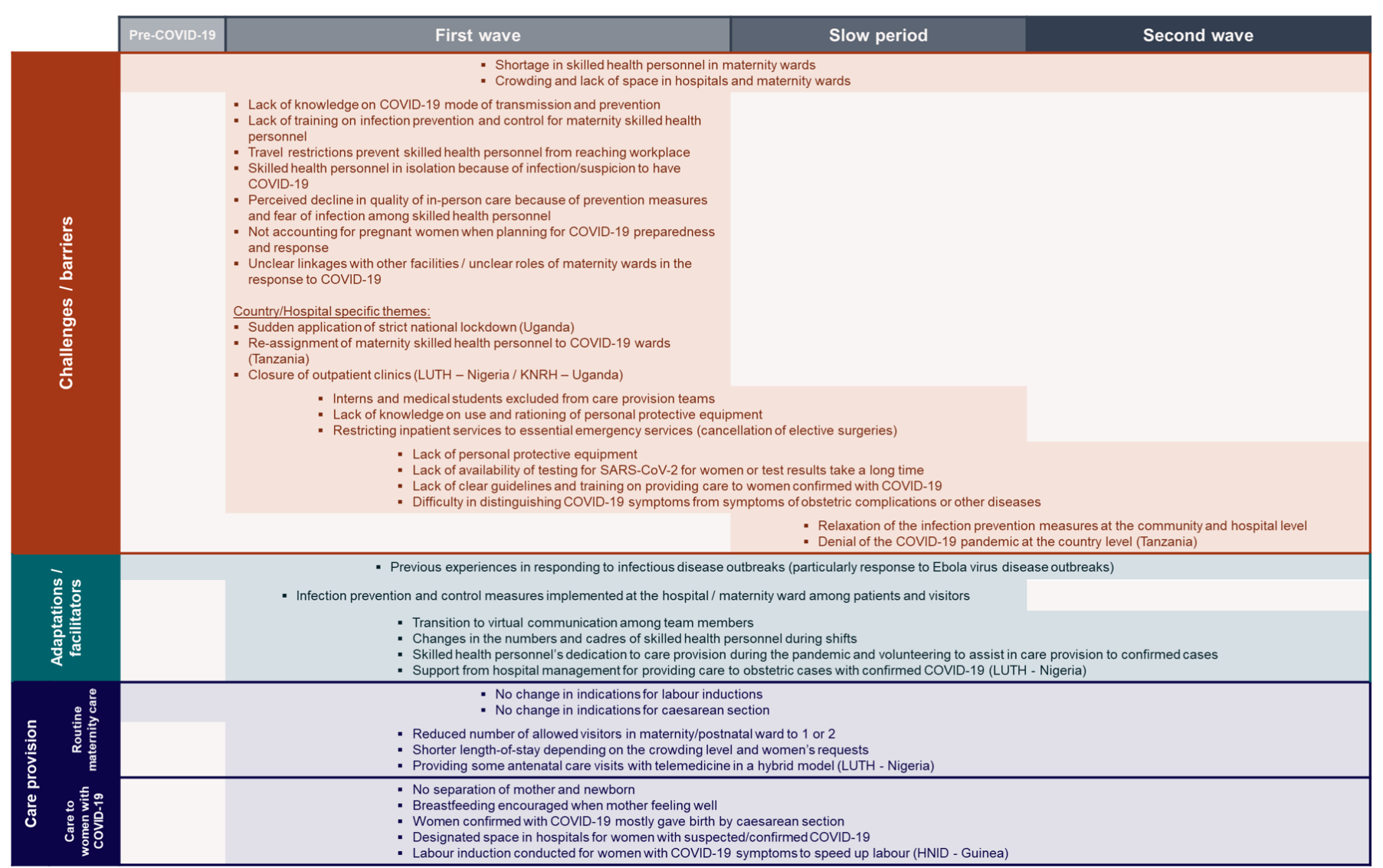

Figure 4 Challenges, adaptations, and care provision before and during the COVID-19 pandemic. HNID, Hôpital National Ignace Deen; KNRH, Kawempe National Referral Hospital; LUTH, Lagos University Teaching Hospital.

The majority of the identified adaptations and facilitators were 'long-term', initiated during the first wave and continued to be applied in the second wave, such as virtual communication between SHP, dedication and commitment of maternity SHP to provide care under difficult circumstances, and support received from the hospital management. The implementation of these adaptations fluctuated dynamically based on resources and epidemiological situation.

\section{DISCUSSION}

This paper explores how maternity care was provided between March 2020 and February 2021, during the first year of the COVID-19 pandemic, in six referral maternity wards in sub-Saharan Africa, using three data sources. Our findings show that the provision of routine maternal care, particularly childbirth care, was generally maintained in these hospitals, despite few interruptions during the first wave. Staffing shortages, lack of PPE, and absence of guidelines and training for maternity staff were some of the factors that led to stress among SHP and contributed to perceived declines in care quality. Nonetheless, maternity wards were able to successfully address most of these challenges during the slow period and second wave. According to SHP, some of the implemented IPC measures negatively affected the quality of care. However, routine procedures and guidelines were mostly preserved in the four countries, and no substantial changes in the proportions of caesarean sections and labour inductions were observed during the COVID-19 pandemic compared with the year before. On a national level, pregnant women were excluded from the response planning early in the pandemic, but the referral hospitals and SHP actively advocated for their inclusion and contributed to guideline development for pregnant women suspected/confirmed with COVID-19. Three of the six hospitals reportedly provided care to pregnant or birthing women confirmed with COVID-19, and they all continued to encourage breastfeeding and were against separation of mother and newborn throughout the study period.

Based on the timeline analysis, the interviews and the discussions with country teams, this paper prospectively documents how challenges, adaptations and facilitators evolved over three time periods. We identified short-term challenges to care provision that were resolved during or just after the first wave. Most of these challenges were linked to the novelty of the disease, such as the little available knowledge about it initially, and the surprise factor resulting from the sudden implementation of restriction measures. Therefore, these initial challenges were easily addressed as the global and local communities became more familiar with COVID-19. In the case of the six hospitals, they also benefited from previous experiences 
with infectious disease outbreaks to overcome these challenges efficiently. Nonetheless, the short duration of these challenges should not undermine their impact on care provision and on the well-being of maternity SHP. Several studies have documented an increase in stress levels, anxiety and depression among frontline workers during the COVID-19 pandemic, and a systematic review shows that PPE shortage, inconsistent information and long working hours are associated with SHP's experiences during pandemics and epidemics globally. ${ }^{28} 29$ Maternity SHP must be included in the priority setting when responding to health system shocks as they constitute a pillar of the WHO's quality of care model. ${ }^{43}{ }^{44}$ Importantly, women and pregnancy should always be included during the early planning and response to health system shocks, including outbreaks.

Long-term or persistent challenges were also identified, and two of them (shortage in SHP and crowding in maternity wards) existed before the COVID-19 pandemic. These are systemic challenges requiring highlevel commitment from health authorities, preparedness, planning and resource availability. Concrete efforts must be put in place to increase staffing levels, alleviate crowding in maternity wards, make rapid testing for COVID-19 available for women in labour and ensure a sustained supply of PPE.

The triangulation of three data sources allowed to visualise how national-level factors coincide with implementing/not implementing adaptations at the hospital level, and how this differed between countries and hospitals. For example, countries' decisions to allocate specific COVID-19 treatment centres can dictate the level of involvement required from maternities in the response, such as the specialisation in treating obstetric cases confirmed with COVID-19, as was the case in Nigeria. ${ }^{32}$ The epidemiology of COVID-19 at the national levels, which was driving decision-making on IPC mandates, was also reflected in the experiences of SHP and their morale over time. The higher the number of diagnosed COVID-19 cases in the community, the higher the chance of having pregnant women confirmed with COVID-19 in the maternity ward. As such, SHP in HRM (Guinea) did not describe any interactions with women confirmed with COVID-19. In Tanzania, the lack of acknowledgement of the disease on a national level was perceived as a difficulty in responding to COVID-19 at MNH (Tanzania), since no clear facility roles or referral systems were implemented, nor were any official guidelines on the management of women confirmed with COVID-19 approved after June 2020.

The cross-country comparison shows how the impact of COVID-19 on provision of maternity care depends on the extent of the epidemiological situation, but most importantly on the pre-existing health system infrastructure and preparedness. For example, in Uganda, SHP faced difficulty in implementing the recommendations due to logistical and resource-related constraints, such as the difficulty in keeping a distance $1.5 \mathrm{~m}$ between women when the maternity wards are crowded and there is not enough space. Country, hospital and ward specificities should be taken into consideration when developing international guidelines and recommendations in response to health system shocks. Voices from low- and middle-income countries should contribute to the guideline development process in a way to ensure that adaptations are achievable within the local circumstances. ${ }^{45}$

Previous experience with the 2014 EVD outbreak is also one of the national-level factors that could have influenced the response during the COVID-19 pandemic. In Guinea, we noted a rapid and efficient revival of response committees which were functioning during the EVD outbreak at the hospital levels. Nationally, a multidisciplinary scientific committee for the COVID-19 responses was established, and this committee was chaired by a woman, a gynaecologist, and two vice-chairmen, an anthropologist and a virologist ${ }^{46}$; which could have contributed to maintaining maternity care as a priority during the pandemic. Additionally, many of the challenges to care provision during the COVID-19 pandemic were common with those experienced during the EVD outbreak, such as travel restrictions, facility closure, exclusion of pregnant women from services and fear among SHP. ${ }^{47}$ Nonetheless, our prospective analysis shows that these challenges did not extend beyond the first wave of COVID-19, possibly showing how health systems in the region have retained lessons from the EVD response and applied them rapidly during the COVID-19 pandemic. Last, in our study, we document how SHP went above and beyond their duties to continue to provide care during the pandemic, and how they volunteered to care for the first women confirmed with COVID-19 in their wards. This dutifulness and sense of responsibility among maternity SHP was also paralleled during the 2014-2016 EVD outbreak in Sierra Leone. ${ }^{48}$

Through this research study, we document key events at the level of the maternity ward that shaped the ability to provide maternal care during the COVID-19 pandemic, and we show how these measures fluctuated over time. Such measures include service closures, referrals to other facilities including COVID-19 treatment centres, new guidelines on changes in visiting rules, on mask wearing mandates, on patients paying PPE costs, among others. These hospital-level mitigation measures were also factors in determining trends in utilisation of maternity healthcare services including antenatal and postnatal care. ${ }^{49}$ Unlike national-level key events for which a number of databases were set up immediately, hospital and maternity ward-level events do not seem to be reported or recorded systematically. The documentation and proper communication of these measures to other health facilities (including lower-level referring facilities and higher-level facilities) and to patients are important for the national coordination of provision of essential healthcare services. We additionally documented gaps in the communication between some maternity wards and COVID-19 treatment centres after referring women 
who were diagnosed with COVID-19. There is a need to enhance these communication channels and bridge the gap in order to ensure that all women receive adequate respectful maternity care.

This is an in-depth description of maternity care provision during the COVID-19 pandemic in four different low-resource settings. The strength of this study lies in the prospective nature which allowed to capture a wide diversity in the pandemic progression, which was unpredictable at the beginning of the research and some elements would have been impossible to capture retrospectively. On the other hand, some limitations of this research include a delay in receiving ethical approval from Uganda, which led us to cover the early wave of the pandemic retrospectively in the interviews, and to recruit only two SHP per maternity ward. The number of respondents may seem small compared with the number of eligible participants in each maternity ward. This is a result of the purposive sampling strategy, aiming to ensure maximum variation in reporting, rather than to ensure representativeness. Additionally, we gathered information from the research team (junior and senior researchers who are clinicians) which was triangulated with the qualitative interviews and aided interpretation. Data on the timeline of COVID-19 were gathered at the national level without disaggregation by regions/districts, although the pandemic spread differently and restrictive measures were not uniformly applied across regions of the same country. Additionally, the international travel ban instituted at the time of data collection meant that in-person field visits were not possible, and the majority of the interviews were conducted online. Nonetheless, the extensive collaboration and team engagement allowed for continuous sharing and iterative discussion of the findings. As a result of the lack of a unified documentation of the numbers of COVID-19 suspected/confirmed obstetric cases, it was challenging for the research team to collect these numbers on a monthly basis. Often these data were not recorded systematically in the hospitals, and were available only informally from PIs or heads of department. Finally, our study did not present women's perspectives on adaptations to care provision and any changes in perceptions of quality of care. Future research exploring women's views is recommended.

\section{CONCLUSION}

This study prospectively documents the evolution of challenges, facilitators and adaptations to maternal care provision in six referrals hospitals in sub-Saharan Africa over the first year of the COVID-19 pandemic. By triangulating three data sources, we identified that hospitallevel adaptations coincided with national-level mitigation measures, with notable differences between countries. We identified short-term challenges to care provision that were resolved during or just after the first wave, and long-term or persistent challenges including some that existed before the start of the pandemic. Systemic challenges to care provision, such as staff shortages and crowding, must be addressed with comprehensive planning, preparedness and commitment of health authorities. This study also documents the indispensable role played by maternity wards in referral hospitals and maternity SHP advocating for the inclusion of pregnant women in the planning and response to the COVID-19 pandemic, including leading and contributing to guideline development and training of lower-level facilities. Essential healthcare services, including maternity care, and frontline healthcare providers must be prioritised in planning and response as the COVID-19 pandemic continues to evolve, and in upcoming healthcare system shocks.

\section{Author affiliations}

${ }^{1}$ Department of Public Health, Institute of Tropical Medicine, Antwerp, Belgium ${ }^{2}$ LSE Health, The London School of Economics and Political Science, London, UK ${ }^{3}$ School of Human Sciences, University of Greenwich, London, UK

${ }^{4}$ Department of Obstetrics and Gynaecology, School of Medicine, College of Health Sciences, Makerere University, Kampala, Uganda

${ }^{5}$ Department of Health Policy Planning and Management, Makerere University, School of Public Health, Kampala, Uganda

${ }^{6}$ College of Medicine, University of Lagos, Akoka, Lagos, Nigeria

${ }^{7}$ Département de Santé Publique, Centre National de Formation et de Recherche en Santé Rurale de Maférinyah, Maférinyah, Forécariah, Guinea

${ }^{8}$ Department of Obstetrics and Gynaecology, Muhimbili University of Health and Allied Sciences, Dar es Salaam, Tanzania, United Republic of

${ }^{9}$ Department of Obstetrics and Gynaecology, Kawempe National Referral Hospital, Kampala, Uganda

${ }^{10}$ Department of Obstetrics and Gynaecology, Mulago Specialized Women and Neonatal Hospital, Kampala, Uganda

${ }^{11}$ Maternité Ignace Deen, Hôpital National Ignace Deen de Conakry, Conakry, Guinea

${ }^{12}$ Maternité, Hôpital Régional de Mamou, Mamou, Guinea

${ }^{13}$ Department of Obstetrics and Gynaecology, Muhimbili National Hospital, Dar es Salaam, Tanzania, United Republic of

${ }^{14}$ Department of Obstetrics and Gynaecology, University of Lagos, Idi-Araba, Lagos, Nigeria

${ }^{15}$ Department of Public Health, Université Gamal Abdel Nasser de Conakry, Conakry, Guinea

Twitter Bosede Bukola Afolabi @Coolgynae and Lenka Benova @lenkabenova

Acknowledgements We would like to thank maternal and newborn healthcare providers who contributed their valuable time to participate in the interviews on repeated occasions despite ongoing difficult circumstances and high workload. We acknowledge Ms Kristien Wynants and Ms Nadine El Rashidi for their support in interview transcription. We also acknowledge all the Institutional Review Committees for providing helpful suggestions on this study protocol, and for the expedited review of this study.

Contributors AS - conceptualisation, data curation, formal analysis, visualisation, writing (original draft preparation), writing (review and editing), and is responsible for the overall content as the guarantor. AB-T—conceptualisation, investigation, data curation, formal analysis, visualisation, writing (original draft preparation), writing (review and editing). OB, ND, AK, SN, 00, MA, WAA, AD, LD, MCD, CM, NM and TS - investigation, data curation, validation, writing (review and editing). TDconceptualisation, funding acquisition, project administration, writing (review and editing). BBA, AN, and AP — conceptualisation, project administration, validation, writing (review and editing). AD—conceptualisation, funding acquisition, project administration, validation, writing (review and editing). LB - conceptualisation, funding acquisition, project administration, methodology, investigation, data curation, formal analysis, writing (original draft preparation), writing (review and editing).

Funding This study was funded by the Institute of Tropical Medicine's COVID-19 Pump Priming fund supported by the Flemish Government-Department of Economy, Science \& Innovation and by the Embassy of the UK in Belgium. LB is funded in 
part by the Research Foundation-Flanders (FW0) as part of her Senior Postdoctoral Fellowship. Research in Guinea was funded by the 4th framework agreement between the Institute of Tropical Medicine and Maférinya Research Centre funded by the Belgian Development Cooperation.

Competing interests None declared.

Patient and public involvement statement Patients and/or the public were not involved in the design, or conduct, or reporting, or dissemination plans of this research.

\section{Patient consent for publication Not required.}

Ethics approval This study involves human participants and was approved by: Institutional Review Board at the Institute of Tropical Medicine in Belgium (ID: 1372/20); Comité National d'éthique pour la Recherche en Santé (CNERS) in Guinea (ID: 058/CNERS/20); Lagos University Teaching Hospital Health Research and Ethics Committee in Nigeria (ID: LUTHHREC/EREV/0520/35); MUHAS Senate Research and Publication Committee (ID: MUHAS-REC-6-2020-282)E; Muhimbili National Hospital Ethics Committee (ID: MNH/IRB///2020/016); National Institute for Medical Research (ID: NIMPR/HQ/R.8a/Vol.IX/3479); Makerere University School of Medicine Research Ethics Committee (ID: 2020-150); Uganda National Council for Science and Technology (ID: HS907ES). Participants gave informed consent to participate in the study before taking part.

Provenance and peer review Not commissioned; externally peer reviewed.

Data availability statement Data are available upon reasonable request. Data sharing agreements will be required prior to making the data available. These data include pseudonymised interview transcripts and aggregate monthly routine data indicators on caesarean section and labour induction. Data requests can be sent to the study PI Professor Lenka Benova at Ibenova@itg.be and the ethics committee at the Institute of Tropical Medicine at irb@itg.be.

Supplemental material This content has been supplied by the author(s). It has not been vetted by BMJ Publishing Group Limited (BMJ) and may not have been peer-reviewed. Any opinions or recommendations discussed are solely those of the author(s) and are not endorsed by BMJ. BMJ disclaims all liability and responsibility arising from any reliance placed on the content. Where the content includes any translated material, BMJ does not warrant the accuracy and reliability of the translations (including but not limited to local regulations, clinical guidelines terminology, drug names and drug dosages), and is not responsible for any error and/or omissions arising from translation and adaptation or otherwise.

Open access This is an open access article distributed in accordance with the Creative Commons Attribution Non Commercial (CC BY-NC 4.0) license, which permits others to distribute, remix, adapt, build upon this work non-commercially, and license their derivative works on different terms, provided the original work is properly cited, appropriate credit is given, any changes made indicated, and the use is non-commercial. See: http://creativecommons.org/licenses/by-nc/4.0/.

\section{ORCID iDs}

Dinah Amongin http://orcid.org/0000-0002-1420-005X

Bosede Bukola Afolabi http://orcid.org/0000-0002-7511-7567

Alexandre Delamou http://orcid.org/0000-0002-9397-7106

\section{REFERENCES}

1 World Health Organization. World Health Organization coronavirus disease (COVID-19) dashboard, 2021. Available: https://covid19. who.int/ [Accessed 19 Oct 2021].

2 Africa CDC. Coronavirus disease 2019 (COVID-19) latest updated on the COVID-19 crises, 2020. Available: africacdc.org/covid-19/ [Accessed 19 Oct 2021].

3 Semaan A, Audet C, Huysmans E, et al. Voices from the frontline: findings from a thematic analysis of a rapid online global survey of maternal and newborn health professionals facing the COVID-19 pandemic. BMJ Glob Health 2020;5:e002967.

4 Kotlar B, Gerson E, Petrillo S, et al. The impact of the COVID-19 pandemic on maternal and perinatal health: a scoping review. Reprod Health 2021;18:10.

5 Khan MSI, Nabeka H, Akbar SMF, et al. Risk of congenital birth defects during COVID-19 pandemic: draw attention to the physicians and policymakers. J Glob Health 2020;10.

6 Khalil A, Kalafat E, Benlioglu C, et al. SARS-CoV-2 infection in pregnancy: a systematic review and meta-analysis of clinical features and pregnancy outcomes. EClinicalMedicine 2020;25:100446.
7 McClure EM, Kinney MV, Leisher SH, et al. Impact of COVID-19 on maternal and child health. Lancet Glob Health 2020;8:e1258.

8 Villar J, Ariff S, Gunier RB, et al. Maternal and neonatal morbidity and mortality among pregnant women with and without COVID-19 infection: the INTERCOVID multinational cohort study. JAMA Pediatr 2021;175:817-826.

9 Nair M, MaatHRI Writing Group, On Behalf Of The MaatHR Collaborators. Reproductive health crisis during waves one and two of the COVID-19 pandemic in India: incidence and deaths from severe maternal complications in more than 202,000 Hospital births. EClinicalMedicine 2021;39:101063.

10 World Health Organization. COVID-19 clinical management - Living Guidance, 2021. Available: https://www.who.int/publications/i/item/ WHO-2019-nCoV-clinical-2021-1

11 Roberton T, Carter ED, Chou VB, et al. Early estimates of the indirect effects of the COVID-19 pandemic on maternal and child mortality in low-income and middle-income countries: a modelling study. Lancet Glob Health 2020;8:e901-8.

12 Chmielewska B, Barratt I, Townsend R, et al. Effects of the COVID-19 pandemic on maternal and perinatal outcomes: a systematic review and meta-analysis. Lancet Glob Health 2021;9:e759-72.

13 World Health Organization. Second round of the National pulse survey on continuity of essential health services during the COVID-19 pandemic: January-March 2021, 2021. Available: https:// www.who.int/publications/i/item/WHO-2019-nCoV-EHS-continuitysurvey-2021.1

14 Wagner M, Falcone V, Neururer SB, et al. Perinatal and postpartum care during the COVID-19 pandemic: a nationwide cohort study. Birth 2021. doi:10.1111/birt.12594. [Epub ahead of print: 07 Oct 2021].

15 Harkness M, Yuill C, Cheyne H, et al. Induction of labour during the COVID-19 pandemic: a national survey of impact on practice in the UK. BMC Pregnancy Childbirth 2021;21:1-8.

16 Townsend R, Chmielewska B, Barratt I, et al. Global changes in maternity care provision during the COVID-19 pandemic: a systematic review and meta-analysis. EClinicalMedicine 2021;37:100947.

17 Okunade KS, Makwe CC, Akinajo OR, et al. Good clinical practice advice for the management of pregnant women with suspected or confirmed COVID-19 in Nigeria. Int J Gynaecol Obstet 2020;150:278-84.

18 Ezenwa BN, Fajolu IB, Akinajo OR, et al. Management of COVID-19: a practical guideline for maternal and newborn health care providers in sub-Saharan Africa. J Matern Fetal Neonatal Med 2020:1-7.

19 World Health Organization. Breastfeeding and COVID-19 - Scientific brief, 2020. Available: https://www.who.int/publications/i/item/WHO2019-nCoV-Sci_Brief-Breastfeeding-2020.1

20 World Health Organization. Maintaining essential health services: operational guidance for the COVID-19 context interim guidance, 2020. Available: https://www.who.int/publications/i/item/WHO-2019nCoV-essential-health-services-2020.1

21 WHO, UNFPA, UNICEF. Continuing essential Sexual, Reproductive, Maternal, Neonatal, Child and Adolescent Health services during COVID-19 pandemic - Practical considerations, 2020. Available: https://apps.who.int/iris/bitstream/handle/10665/332162/RG Detailed_-eng.pdf?sequence $=1$ \&isAllowed $=y$

22 Rimmer MP, Al Wattar BH, Members U, UKARCOG Members. Provision of obstetrics and gynaecology services during the COVID-19 pandemic: a survey of junior doctors in the UK National health service. BJOG 2020;127:1123-8.

23 Szabo RA, Wilson AN, Homer C, et al. COVID-19 changes to maternity care: experiences of Australian doctors. Aust N Z J Obstet Gynaecol 2021;61:408-15.

24 Huysmans E, Audet C, Delvaux T. How COVID-19 challenged care for women and their newborns: a qualitative case study of the experience of Belgian midwives during the first wave of the pandemic. medRxiv 2021;052121257440:2021.

25 Rao SPN, Minckas N, Medvedev MM, et al. Small and sick newborn care during the COVID-19 pandemic: global survey and thematic analysis of healthcare providers' voices and experiences. BMJ Glob Health 2021;6:e004347.

26 Ameh C, Banke-Thomas A, Balogun M, et al. Reproductive maternal and newborn health providers' assessment of facility preparedness and its determinants during the COVID-19 pandemic in Lagos, Nigeria. Am J Trop Med Hyg 2021;104:1495-506.

27 Bradfield Z, Wynter K, Hauck Y, et al. Experiences of receiving and providing maternity care during the COVID-19 pandemic in Australia: a five-cohort cross-sectional comparison. PLoS One 2021;16:e0248488. 
28 Chigwedere OC, Sadath A, Kabir Z, et al. The impact of epidemics and pandemics on the mental health of healthcare workers: a systematic review. Int J Environ Res Public Health 2021;18:6695.

29 Afulani PA, Nutor JJ, Agbadi P, et al. Job satisfaction among healthcare workers in Ghana and Kenya during the COVID-19 pandemic: role of perceived preparedness, stress, and burnout. PLOS Glob Public Health 2021;1:e0000022.

30 Stein DT, Golub G, Rothschild CW, et al. Bypassing high-quality maternity facilities: evidence from pregnant women in peri-urban Nairobi. Health Policy Plan 2021;36:84-92.

31 Kruk ME, Hermosilla S, Larson E, et al. Bypassing primary care clinics for childbirth: a cross-sectional study in the Pwani region, United Republic of Tanzania. Bull World Health Organ 2014;92:246-53.

32 Ishola O. Giving hope to pregnant COVID-19 patients: LUTH in giant strides, 2020. Available: https://www.vanguardngr.com/2020/07/ giving-hope-to-pregnant-covid-19-patients-luth-in-giant-strides/

33 Kumari V, Mehta K, Choudhary R. COVID-19 outbreak and decreased hospitalisation of pregnant women in labour. Lancet Glob Health 2020;8:e1116-7.

34 Ashish KC, Peterson SS, Gurung R, et al. The perfect storm: disruptions to institutional delivery care arising from the COVID-19 pandemic in Nepal. J Glob Health 2021;11:05010.

35 Shapira G, Ahmed T, Drouard SHP, et al. Disruptions in maternal and child health service utilization during COVID-19: analysis from eight sub-Saharan African countries. Health Policy Plan 2021;36:1140-51.

36 Soma-Pillay P, Moodley J, Pattinson R. The effect of the first wave of COVID-19 on use of maternal and reproductive health services and maternal deaths in South Africa. Obstetrics and gynaecology forum, 2020:38-46.

37 Kc A, Gurung R, Kinney MV, et al. Effect of the COVID-19 pandemic response on intrapartum care, stillbirth, and neonatal mortality outcomes in Nepal: a prospective observational study. Lancet Glob Health 2020;8:e1273-81.

38 Creswell JW, Clark VLP. Choosing a mixed method design. Designing and conducting mixed methods research. SAGE publications, 2011: 53-107.
39 Hale T, Angrist N, Goldszmidt R, et al. A global panel database of pandemic policies (Oxford COVID-19 government response Tracker). Nat Hum Behav 2021;5:529-38.

40 University of Oxford. Our World in Data - Statistics and Research - Coronavirus (COVID-19) Deaths, 2021. Available: https:// ourworldindata.org/covid-deaths

41 Gale NK, Heath G, Cameron E, et al. Using the framework method for the analysis of qualitative data in multi-disciplinary health research. BMC Med Res Methodol 2013;13:117.

42 Salyer SJ, Maeda J, Sembuche S, et al. The first and second waves of the COVID-19 pandemic in Africa: a cross-sectional study. Lancet 2021;397:1265-75.

43 Wilson AN, Ravaldi C, Scoullar MJL, et al. Caring for the carers: ensuring the provision of quality maternity care during a global pandemic. Women Birth 2021;34:206-9.

44 World Health Organization. WHO recommendations: intrapartum care for a positive childbirth experience, 2018. Available: https:// www.who.int/reproductivehealth/publications/intrapartum-careguidelines/en/

45 Maaløe N, Ørtved AMR, Sørensen JB, et al. The injustice of unfit clinical practice guidelines in low-resource realities. Lancet Glob Health 2021;9:e875-9.

46 Bonnet E, Bodson O, Le Marcis F, et al. The COVID-19 pandemic in francophone West Africa: from the first cases to responses in seven countries. BMC Public Health 2021;21:1-17.

47 Yerger $\mathrm{P}$, Jalloh M, Coltart CEM, et al. Barriers to maternal health services during the Ebola outbreak in three West African countries: a literature review. BMJ Glob Health 2020;5:e002974.

48 Drevin G, Mölsted Alvesson H, van Duinen A, et al. "For this one, let me take the risk": why surgical staff continued to perform caesarean sections during the 2014-2016 Ebola epidemic in Sierra Leone. BMJ Glob Health 2019;4:e001361.

49 Banke-Thomas A, Semaan A, Amongin D. A mixed-methods study of maternal health care utilisation in six referral hospitals in four sub-Saharan African countries before and during the COVID-19 pandemic. BMJ Global Health 2021. 